\title{
LA INVESTIGACIÓN SOBRE EDUCACIÓN TÉCNICO PROFESIONAL DE NIVEL MEDIO EN ARGENTINA Y BRASIL A PARTIR DE LOS '90. PRIMERAS APROXIMACIONES AL ESTADO DEL ARTE'.
}

\author{
Yanina D. Maturo ${ }^{2}$
}

\section{RESUMEN}

Este artículo se propone sistematizar a modo de una primera aproximación al "estado del arte", las investigaciones y estudios sobre Educación Técnico Profesional (ETP) de nivel medio en Argentina y Brasil a partir de la década de los '90 hasta nuestros días. El recorte temporo espacial se realizó atendiendo a los requerimientos de la investigación en curso y al reconocimiento de los cambios significativos por los que ha atravesado la ETP en los últimos 20 años en ambos países y que ha llevado a posicionarla como objeto de debate en las agendas políticas nacionales e internacionales, dando como resultado numerosos estudios e investigaciones al respecto.

Palabras Clave: educación técnico profesional; nivel medio; estado del arte.

\section{A PESQUISA SOBRE A EDUCAÇÃO PROFISSIONAL Y TECNOLÓGICA (ETP) DE NÍVEL MÉDIO EM ARGENTINA E BRASIL A PARTIR DO INÍCIO DOS ANOS '90. PRIMEIRAS ABORDAGENS AO ESTADO DA ARTE.}

\section{RESUMO}

Este trabalho pretende sistematizar por meio de uma primeira abordagem ao " estado da arte" as pesquisas e estudos sobre a Educação Profissional y Tecnológica (ETP) de nível médio de Argentina e Brasil a partir do início dos anos 90 até os dias atuais. O corte espacial temporo foi realizada de acordo com os requisitos da pesquisa e do reconhecimento de que as alterações significativas que passou a ETP, nos últimos 20 anos, em ambos países e levou a posicioná-lo como tema de discutido nas agendas políticas nacionais e internacionais, resultando em inúmeros estudos e investigações.

Palavras-chave: educação profissional técnica; nível médio; estado da arte

\section{PRESENTACIÓN}

La construcción del estado del arte es una etapa sustancial en el proceso de investigación que aporta a un conocimiento más acabado de la temática, posibilitando delimitar la problemática de manera más precisa, inspeccionando sobre lo no abordado por otros investigadores con el objetivo de establecer nuevos caminos metodológicos y perspectivas teóricas que aporten nuevas formas de abordaje. Como afirma Jiménez Becerra, "sólo se problematiza lo que se conoce, y para conocer y problematizar un objeto de estudio es necesaria una aprehensión inicial por lo ya dado, en este caso, el acumulado investigativo, condensado en diferentes textos e investigaciones que antecedieron a mi inclinación temática” (2006:32). 
En este sentido, este artículo se propone sistematizar a modo de una primera aproximación al "estado del arte", las investigaciones y estudios sobre Educación Técnico Profesional (ETP) de nivel medio en Argentina y Brasil a partir de la década de los '90 hasta nuestros días. El recorte temporo espacial se realizó atendiendo a los requerimientos de la investigación en curso y al reconocimiento de los cambios significativos por los que ha atravesado la ETP en los últimos 20 años en ambos países y que ha llevado a posicionarla como objeto de debate en las agendas políticas nacionales e internacionales, dando como resultado numerosos estudios e investigaciones al respecto.

La información recolectada fue organizada en función de cuatro líneas de investigación relevantes: a) la relación entre educación y trabajo, b) la perspectiva de las políticas educativas, c) los aspectos pedagógicos y didácticos $\mathrm{y}$, finalmente, d) la perspectiva comparada entre Argentina y Brasil.

Las fuentes que se consultaron para la realización de este trabajo corresponden a bases de datos de universidades públicas, red Scielo y Redalyc; lo que arrojó como resultado el relevamiento de cuarenta investigaciones, veinte por cada país, muchas de las cuales remiten preferentemente a trabajos de maestría y doctorado. Asimismo, se recuperaron los trabajos de investigadores independientes de larga trayectoria en el estudio de la ETP como María Antonia Gallart de Argentina y Gaudencio Frigotto de Brasil. Por último, se presentan diez investigaciones que incorporan un enfoque comparado entre Argentina y Brasil sobre la ETP.

\section{LA INVESTIGACIÓN SOBRE ETP EN ARGENTINA Y BRASIL: ENFOQUES Y PERSPECTIVAS.}

En los últimos 20 años, tanto Argentina como Brasil han sido testigos de grandes transformaciones en el mundo de la economía, la tecnología y el empleo. La formación para el mundo del trabajo se tornó en una preocupación de gran relevancia en ambos países, por lo cual se pusieron en marcha importantes reformas en el sistema de enseñanza que intentaron incrementar la cobertura de la educación formal y preparar mejor a los jóvenes para su futura inserción en el mundo del trabajo ${ }^{3}$.

En este contexto se desarrollaron un conjunto de investigaciones que intentaron comprender -pero a la vez también dar respuestas- a las transformaciones que se venían dando en la ETP de nivel medio como la expresión más tangible de lo que era la educación para el trabajo. Estas investigaciones han abordado desde diferentes perspectivas el estudio de la ETP, pudiéndose evidenciar cuatro líneas de indagación relevantes: a) la relación entre educación y trabajo, b) la perspectiva de las políticas educativas, c) los aspectos pedagógicos y didácticos y, finalmente, d) la perspectiva comparada entre Argentina y Brasil.

Con respecto a la primer línea de investigación, la relación entre educación y trabajo, se encontraron en Argentina un conjunto de estudios sobre la modalidad de la ETP de nivel medio que se caracterizan por realizar una reconstrucción histórica de la relación entre educación y trabajo, remarcando su "función económica" como marca distintiva.

El análisis de la relación escuela y sector productivo resulta insoslayable en estas investigaciones para poder entender las modificaciones en las funciones y objetivos de la modalidad a través de la historia. Algunas de estas producciones enfocan su análisis en la gestión del gobierno peronista (1946-1955), destacándolo como el período de auge de la ETP en Argentina en tanto oportunidad de ascenso social para la clase más desfavorecida (Puiggrós \& Bernetti, 1993; Pineau, 1997; Dussel \& Pineau, 2003). Consideran como 
nefastos los efectos de las políticas neoliberales de los años '90 y "desarticuladoras" de la oferta de ETP en el país, eximiendo al Estado de su responsabilidad educativa y prevaleciendo la lógica empresarial, donde el mercado es el nuevo encargado de valorar y legitimar los conocimientos en tanto "competencias evaluables" por parte de las empresas (Sobrevila, 1995; Gallart, 2002; Garcés, 2007; Spinosa \& Testa, 2009).

En lo que refiere a Brasil, también se encontraron trabajos que -centrando su análisis en la relación educación y trabajo desde una perspectiva histórica- pretenden contribuir al campo de la ETP. Son investigaciones que se enfocan en la evolución de la ETP desde "la colonia" hasta las últimas décadas, destacando su articulación con el sector productivo y los condicionamientos del contexto socio, político y cultural de cada período (Silva, 2004; Da Cruz, 2008; Carvalho, 2008). Algunos de estos trabajos subrayan la necesidad de superar la dualidad entre educación general y educación profesional, problemática que acarrea históricamente el sistema de formación profesional de nivel medio en Brasil y que tiene implicancias determinantes en las necesidades de formación profesional de los alumnos (Poli, 1999). Son estudios que integran un enfoque histórico al planteo de problemáticas que llevan larga data en el sistema educativo brasilero y que están estrechamente vinculadas a los cambios en el sistema de producción.

El análisis de las políticas educativas para la modalidad como objeto de estudio, también ha sido abordado por distintos académicos de ambos países. Se pudieron identificar dos grandes períodos estudiados: la década de los '90 y los primeros años del siglo XXI.

En lo que respecta al primer período identificado, se encontraron trabajos en Argentina que analizan los efectos de los cambios normativos sustentados por la Reforma Educativa de los '90 y sus efectos en las históricas escuelas técnicas de nivel medio (Steinkol \& Croce, 2001; Testa \& Sánchez, 2003; Gallart, 2003; Alterman, 2005; Abratte \& Pacheco, 2006; Gallard, 2006; Maturo \& Rubio, 2008).

Estas investigaciones subrayan las modificaciones introducidas por la Reforma de los '90 -sobre todo en lo que respecta a la organización institucional, al currículum y al impacto en el trabajo docente- y cómo la misma llevó a poner en riesgo la propia existencia de la escuela técnica. Se destaca la transferencia de los servicios educativos de nivel medio y la imposición de un nuevo currículum como elementos claves en el análisis de las situaciones que llevaron a reconfigurar tanto la oferta educativa como el trabajo diario en las escuelas. Enfatizan el rol de las instituciones educativas en el proceso de recontextualización y re-significación de la normativa, asignándole a los equipos directivos y de docentes un rol activo y estratégico en la puesta en práctica del nuevo marco normativo, sobre todo en lo referente al resguardo de las funciones propias de la modalidad ante los cambios que planteaba la reforma.

En Brasil las políticas educativas desarrolladas durante la década de los '90 para la modalidad de la ETP, coincidentemente con el caso argentino, han sido un importante foco de análisis. Se relevaron trabajos que ponen especial énfasis en la función y objetivos de la educación profesional de nivel medio durante ésta década analizando las implicancias de las políticas neoliberales y los efectos en la configuración de la oferta; lo cual se vio plasmado en lo que los autores denominaron "la era de las directrices", haciendo referencia al conjunto de regulaciones (políticas) emanadas desde el gobierno nacional para organizar el sistema de enseñanza (Frigotto \& Ciavatta 2003, Ferretti, 2009, Ciavatta \& Ramos 2012). Los marcos normativos creados durante éste período son considerados por los investigadores como un "retroceso" en materia institucional, organizativa y pedagógica de la educación básica brasilera en general y de la ETP en particular. Asimismo, algunos remarcan la necesidad de pensar un cambio estructural a nivel social que lleve a re-pensar 
una educación de calidad que fomente la igualdad de oportunidades y la inclusión de los más pobres.

En relación al estudio del desarrollo y puesta en marcha de políticas educativas para la ETP durante los primeros años del siglo XXI, en esta primera revisión bibliográfica no se visualizaron producciones de relevancia para el caso argentino. Por el contrario, en Brasil, se encontraron investigaciones que analizan profundamente cuestiones referidas a las políticas educativas desplegadas para la re-organización, democratización y financiamiento de la ETP.

Algunos de estos estudios se centran específicamente en el análisis de la gestión y organización de la modalidad a partir de la sanción de un nuevo marco legal durante el gobierno de Luiz Inácio Lula da Silva (Ortigara, 2012); otros, resaltan el impacto de las políticas curriculares desarrolladas para la ETP en su conjunto y en lo que refiere a su articulación con otras modalidades del sistema (educación de jóvenes y adultos PROEJA $^{4}$ ) (Guimarães, 2008; da Silva, 2011; Cremonese, 2012). También se destacan investigaciones que hacen foco en el análisis de la dinámica público-privado y el rol del Estado en la regulación de la oferta de ETP (Costa Gomes, 2008) y, por último, se visualizan estudios que analizan el controvertido proceso de aplicación de esta nueva propuesta normativa en relación a las demandas sociales, las acciones concretas desarrolladas desde el gobierno y las omisiones en el ejercicio de poder en dicho proceso (Frigotto, Ciavatta \& Ramos, 2005).

En general, estos estudios realizan un análisis sobre las características de organización y funcionamiento de la modalidad en estos primeros años del siglo XXI; que si bien los consideran de re-estructuración de la ETP, no dejan de cuestionar algunos mecanismos de regulación de la nueva política sobre las instituciones: estructura, organización, financiamiento, currículum y sistemas de evaluación (Brezinski, 2011; Brenzinski \& Aguilar, 2013).

Sobre la tercera línea de investigación, aspectos pedagógicos y didácticos, se hallaron algunos trabajos de diferentes autores argentinos que destacan la formación en competencias como tema central a la hora de pensar las funciones y objetivos de la modalidad de la ETP (Novick, Bartolome, Buceta y otras, 1998; Briasco \& Vargas, 2002; Gallart, 2004; Jacinto, 2004, 2010; Jacinto \& Millenar, 2007). Estas investigaciones subrayan la necesidad de detectar las demandas de competencias del sector productivo y las respuestas reales de las instituciones educativas a dichas demandas. Consideran necesario atender la relación entre demanda de competencias y ofertas de capacitación como punto de partida para fortalecer la relación escuela y trabajo. También en Brasil, se encontraron trabajos que abordan la formación en competencias a la hora de analizar la utilidad y eficacia de la formación profesional como respuesta a las demandas del mercado (Gonçalves, 2001; Mendes, 2005; Eslabão; 2006; Frigotto, 2007).

Por último, se consideraron de interés 10 trabajos que aportan al análisis comparado de la modalidad de la ETP entre Argentina y Brasil. En una primera revisión, se identificaron algunos estudios que se caracterizan por ser informes de organismos o agencias internacionales (OIT-CINTERFOR ${ }^{5}, \mathrm{CEPAL}^{6}$, IIPE-UNESCO ${ }^{7}$ ), realizados por especialistas de diversos países (Ibarrola \& Gallart, 1994; Garmendia Arigón, 2000; Briasco \& Baldes 2000; Briasco, 2005, 2008; Finnegan, 2006; Velasco, 2007). Estos estudios aportan información global sobre el estado o la situación de la temática (revisión de matrícula, estructura y organización del sistema, relación con el sistema productivo, etc.) y en muchos casos proponen líneas de acción y formulan sugerencias ${ }^{8}$.

Entre las producciones locales que incorporan una perspectiva de estudio comparado encontramos el trabajo de Melo (2009) y el de Delphino (2009, 2010), las 
cuales centran su análisis en las políticas educativas de Argentina y Brasil para la ETP. En el primer caso, la autora analiza el impacto de las políticas educativas durante la década de los'90 en el trabajo de los docentes de escuelas de ETP, determinando las acciones de adhesión y/o resistencia a la política educativa por parte de los docentes y las manifestaciones de conflictos que surgieron a partir de las transformaciones en la organización escolar y la organización del trabajo en dicho período. En el segundo caso, Delphino $(2009,2010)$ estudia las políticas públicas desarrolladas en los últimos cuarenta años en Brasil y Argentina, centrándose en la relación entre los planes económicos y proyectos educativos para la educación técnico profesional; analizando cómo la política educativa en cada país ha tratado de responder a los requerimientos del mercado sin dar los resultados esperados.

Ambos trabajos realizan aportes interesantes para comprender las similitudes y diferencias en la organización y administración del subsistema de ETP desde sus orígenes hasta nuestros días. En palabras de las autoras, la cuestión social y política de cada país en diferentes momentos históricos resulta imprescindible para comprender dichos procesos y los cambios en la relación educación y trabajo y el papel de la ETP en esta encrucijada.

\section{Acerca de opciones teóricas y metodológicas...}

Se pudo observar que la ETP ha sido y es una preocupación de las agendas nacionales e internacionales de gobierno y esto se expresa en los estudios que se han llevado a cabo abordando diferentes dimensiones de la misma y tratando de buscar posibles explicaciones a las transformaciones acontecidas en el último tiempo.

Si bien la extensión de este artículo no permite explayarse demasiado, resulta interesante realizar algunas consideraciones sobre los enfoques, perspectivas teóricas metodológicas y alcances que se pueden evidenciar como opciones de investigación en las producciones mencionadas.

Se ha podido identificar que la totalidad de los estudios citados remiten a un enfoque de tipo cualitativo, aunque no descartan la incorporación de datos cuantitativos que optimizan la triangulación de información. También podemos decir que la recolección de datos se basa sobre todo en el análisis bibliográfico para los estudios donde prevalece una perspectiva histórica y descriptiva y, la realización de entrevistas y revisión de legislación en aquellas investigaciones que pretenden un alcance más explicativo e interpretativo de los procesos de transformación de la ETP y su realidad.

La estrategia metodológica adoptada por los investigadores en estos estudios remite preferentemente al estudio de caso, en tanto permite el análisis de fenómenos de la actualidad con el fin de proporcionar herramientas, como conceptos teóricos, que ayuden a comprender o solucionar el problema; por lo general, estos estudios también parten de situaciones hipotéticas o supuestos que a través del avance del proceso de investigación son "corroborados" o "desechados". En palabra de los autores "los resultados pretenden generar explicaciones certeras o soluciones viables sobre el fenómeno en estudio".

En lo que refiere a la adopción de una perspectiva epistemológica (en tanto cosmovisión que el investigador asume para guiar su investigación) se pudieron detectar ciertas diferencias entre las investigaciones relevadas en Argentina y Brasil. En el caso de las investigaciones de Argentina, sólo en un trabajo se puedo identificar la explicitación del uso de algunas categorías teóricas del marxismo -como Teoría General- para el estudio realizado, lo que derivó en un posicionamiento dialéctico sobre la construcción de la historia de la educación. En los demás trabajos sólo se expone una lista de autores que se utilizan como marco de referencia pero no hay una justificación epistemológica y 
ontológica de dicha selección y muchas veces no guardan coherencia entre los mismos. Es decir, existe una ausencia de explicitación por parte del investigador de las presuposiciones o premisas que sostiene acerca de cómo leer la realidad e interpretar el mudo (cosmovisión).

Por el contrario, en Brasil, algunos trabajos explicitan con claridad el posicionamiento adoptado por el investigador. En siete de los veinte trabajos revisados, los investigadores adhieren y enmarcan su trabajo en una perspectiva (o Teoría General) marxista o neo-marxista posicionándose desde un enfoque crítico-dialéctico (Teoría Sustancial), y justifican la metodología de trabajo en base a estos presupuestos teóricos. En este sentido, los autores recuperan con agudeza los trabajos de los clásicos del materialismo histórico-dialéctico: Karl Marx, Friedrich Engels y Antonio Gramsci; como así también, recuperan otros autores locales que siguen la misma línea de análisis, como es el caso de: Demerval Saviani y Gaudencio Frigotto.

En el resto de los trabajos, si bien no se logra vislumbrar un claro posicionamiento de los investigadores, se evidencia la presencia de los clásicos más arriba mencionados y de algunas de sus categorías teóricas-analíticas, a lo cual se suma el aporte de otros pensadores como Althuser, Bernstein y Apple.

\section{ALGUNAS CONSIDERACIONES SOBRE LOS ORGANISMOS INTERNACIONALES Y SUS APORTES PARA LA ETP EN AMÉRICA LATINA.}

Como ya se mencionó en el apartado anterior, distintos organismos internacionales como la OIT, la UNESCO, la CEPAL y el Sector Educativo del MERCOSUR han generado espacios de trabajo e investigación conjunta entre especialistas a fin de indagar sobre el campo de la ETP y sus contribuciones al desarrollo de los países de América Latina.

Las implicancias de dichos informes van mucho más allá de una mera indagación sobre la realidad y situación de la ETP en los países Latinoamericanos sino que forman "discursos" "que son presentados por estos organismos como "recomendaciones" o "sugerencias" que terminan traduciéndose en los textos políticos que desarrollan los países para la ETP.

Hace ya varias décadas la $\mathrm{ONU}^{10}$, a través de la celebración de diferentes cumbres y reuniones, se encargó de promover una "conciencia mundial" sobre la necesidad de elaborar informes para evaluar el equilibrio entre los objetivos económicos y los objetivos de desarrollo social de los diferentes países; tendencia que ha sido adoptada por distintas organizaciones preocupadas por la educación y que es traducida en proyectos estadísticos internacionales (Neves, 2008). En este marco, la educación para el trabajo -a través de la ETP- ha sido considerada como el medio por el cual los países pueden alcanzar el máximo desarrollo económico y social (Ferreira \& Paissé, 2003).

La UNESCO ha considerado la ETP como instrumento esencial para la superación de la pobreza en el marco del Acuerdo de "Educación Para Todos (EPT)", compromiso mundial asumido en Dakar durante el año 2000. Por medio de la OREALC ${ }^{11}$, el PREALC $^{12}$ y el IIPE lleva a cabo diversas actividades como la cooperación en la transición de los jóvenes de la escuela al mundo del trabajo, los baremos mundiales de referencias, la definición de la agenda post-2015 sobre educación y desarrollo de competencias profesionales, además de los foros regionales y conferencias internacionales. 
La OIT, por su parte, promueve la justicia social, los derechos humanos y laborales reconocidos a nivel internacional. En la actualidad, favorece la creación de un trabajo decente y las condiciones laborales y económicas que permitan a trabajadores y empleadores su participación en el ámbito económico y social. A través del CINTERFOR gestiona la construcción colectiva de conocimiento y la promoción de la cooperación entre los países en temas relacionados con el desarrollo de los recursos humanos, poniendo especial énfasis en la ETP.

Desde el Sector Educativo del MERCOSUR, también se vienen fomentando procesos de integración regional que, si bien son incipientes, tienden a conformar un espacio educativo común a través de la concertación de políticas que estimulen la movilidad, el intercambio y la formación de una identidad y ciudadanía regional. Estas acciones han impactado de lleno en los países que lo integran y han llevado a la creación de importantes acuerdos e instancias de discusión para la integración educativa en los diferentes niveles de enseñanza, involucrando la ETP. Esta necesidad de definir líneas de acción y crear un "discurso común" sobre la educación para el trabajo y las funciones que deben cumplir las instituciones de ETP en la región, ha derivado también en una demanda creciente de estadísticas e indicadores que permitan evaluar la situación de los países miembros, en lo que respecta el mundo del trabajo y la formación profesional.

Dentro de la estructura del MERCOSUR existen órganos que se dedican específicamente al análisis de la situación del mercado laboral en los diferentes países, esos organismos son: Subgrupo de trabajo No 10 de "Relaciones Laborales, Empleo y Seguridad Social (SGT10)", Comisión Socio-laboral del MERCOSUR (CSL), Grupo de alto Nivel de Empleo (GANENPLE) y el Observatorio del Mercado de Trabajo del MERCOSUR (OMTM).

Los estudios que se elaboran desde estos órganos del MERCOSUR, informan sobre la situación del mercado laboral de los países miembros para luego repensar las políticas de ETP que permitan mejorar la formación de la población para su inserción en el mercado de trabajo y contribuir así a la integración económica regional. Hacen especial énfasis sobre la situación de los jóvenes en el mercado de trabajo, en lo que refiere a la participación laboral, nivel de empleo, nivel de estudio, pobreza, etc.; tratando de identificar las principales tendencias del empleo juvenil para recopilar las medidas políticas laborales para la población y proveer elementos para evaluar las posibles líneas de acción a nivel regional.

Ahora bien, en general los informes emanados desde estas distintas organizaciones (OIT, UNESCO, MERCOSUR, etc.) involucran un conjunto de indicadores que sirven como referencia para el análisis del avance de las políticas educativa para el sector de la ETP y la futura toma de decisiones por parte de las agencias gubernamentales. Ofrecen datos preferentemente cuantitativos que pretenden aportar a una descripción, caracterización y diagnóstico del sistema de enseñanza de la ETP para luego realizar "recomendaciones" o "sugerencias" que permitan superar las problemáticas detectadas y colaborar en la redefinición de las políticas educativas para el sector ${ }^{13}$.

Se puede vislumbrar que la presentación de la información que se realiza desde estos organismos posee una organización común que nos permiten arriesgar un "modelo" que se elabora y estructura en base a determinados indicadores educativos comparables y cuantificables que son organizados generalmente bajo algún esquema sistémicoestratégico; es decir, de acuerdo a los objetivos y metas planteadas en los proyectos políticos-institucionales que se sostienen desde dichos organismos (Taccari, 2011): 


\begin{tabular}{|c|c|}
\hline DIMENSIONES & INDICADORES \\
\hline $\begin{array}{l}\text { Desarrollo económico y bienestar social del } \\
\text { país en estudio. }\end{array}$ & 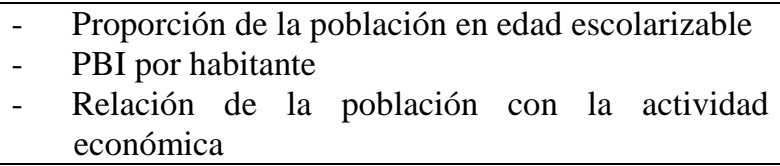 \\
\hline Escolarización y procesos educativos (ETP) & $\begin{array}{ll}\text { - } & \text { Evolución de las tasas de escolarización en la } \\
\text { modalidad } \\
\text { - } & \text { Desarrollo de competencias. } \\
\text { - } & \text { Acceso al nivel superior } \\
\text { - } & \text { Reformas curriculares } \\
\text { - } & \text { Políticas educativas para el sector }\end{array}$ \\
\hline Optimización de recursos (ETP) & $\begin{array}{ll}\text { - } & \text { Gasto público en educación } \\
\text { - } & \text { Gasto en infraestructura y equipamiento } \\
\text { - } & \text { Perfeccionamiento y capacitación de directivos y } \\
\text { docentes } & \text { Integración de las TICs }\end{array}$ \\
\hline Resultados educativos (ETP) & $\begin{array}{ll}\text { - } & \text { Tasas de ingreso a la modalidad } \\
\text { - } & \text { Tasas de abandono en la modalidad } \\
\text { - } & \text { Tasas de egreso en la modalidad } \\
\text { - } & \text { Inserción en el mundo laboral }\end{array}$ \\
\hline
\end{tabular}

Los objetivos de los informes tienen que ver preferentemente con aportar al entendimiento, seguimiento y evaluación de las políticas educativas en el marco de proyectos políticos - institucionales específicos para la ETP.

Asimismo, las conclusiones a las que se llega a través de estos estudios, que en su mayoría utilizan como estrategia de análisis la comparación de casos, no contemplan factores vinculados al contexto del proceso educativo, a cómo se desarrolla dicho proceso de acuerdo a las influencias del medio social; lo que es de suma importancia para comprender los resultados educativos. De ello surge una lectura que podríamos considerar de carácter lineal y acotada de los procesos educativos. Se observa "una duplicación de esfuerzos" y "la presentación de información distinta para procesos y variables iguales", lo que deviene de la ausencia de una adecuada cooperación interinstitucional entre estos organismos para acordar un sistema de indicadores común Taccari (2011).

\section{CONCLUSIONES}

En el marco de la investigación que se está realizando esta aproximación al estado del arte ha pretendido dar una mirada general sobre lo estudiado acerca de la ETP tanto en Argentina como en Brasil desde la década de los '90 hasta nuestros días.

Se ha podido evidenciar de esta manera que el campo de investigación de la ETP es fecundo y que son numerosos los agentes y agencias que intervienen en su crecimiento, desde organismos internacionales hasta prestigiosos investigadores del ámbito académico

La ETP ha sido estudiada desde diferentes dimensiones y a través de distintas metodologías y estrategias de análisis. Los trabajos realizados en Brasil sobre la ETP están brindando un interesante aporte al campo a través del uso de determinadas perspectivas epistemológicas que nos invitan a pensar la ETP en relación a cuestiones que tienen que ver con la división social del trabajo, la lucha de clases y el poder político.

En Argentina, en las investigaciones revisadas, no se ha logrado comprobar un claro posicionamiento epistemológico adoptado por los investigadores; sin embargo, se evidencia un esfuerzo por entender los efectos que han producido y que producen los cambios sociales y políticos en la organización y administración de la ETP en las últimas décadas y cómo ello afecta la propuesta de enseñanza de la modalidad. 
Por otra parte, los aportes de los organismos internacionales pueden resultar interesantes insumos para las instancias de elaboración y desarrollo de las políticas educativas para la ETP por parte de los gobiernos, e igualmente para re-pensarlas; no obstante, los resultados presentados hasta ahora no alcanzan para realizar un análisis profundo de las problemáticas que se plantean en el nivel de la práctica y sólo se quedan en el análisis superficial de cambios visibles o estructurales.

Asimismo, también cabe mencionar que la utilización del estudio comparado, en el marco de los procesos de internacionalización y regionalización de la educación que se están evidenciando, puede resultar una estrategias potente de análisis que aporte a un entendimiento más acabado de los cambios que se suceden a nivel macro y que impactan inevitablemente en el ámbito de lo local. Resulta imprescindible empezar a mirarnos en relación al otro no sólo para evidenciar diferencias y similitudes sino también para poder explicarlas y buscar soluciones a las problemáticas actuales.

Por último, es preciso remarcar la idea que traen Tello \& Mainardes (2012) - desde una concepción de no neutralidad de los investigadores en la producción de conocimiento-, sobre la necesidad de explicitar la perspectiva y posicionamiento epistemológico en los procesos de investigación, como modo de ejercer una "vigilancia epistemológica" que permite y a la vez garantiza estar atentos ante ciertas incoherencias o contradicciones en las diferentes etapas del proceso. Ello nos previene de no caer en una visión positivista de las ciencias o el desconocimiento de perspectivas epistemológicas que pueden resultar muy útiles a nuestros objetivos de investigación.

\section{BIBLIOGRAFÍA}

ABRATTE, J. P. \& PACHECO, M. (2006). La escuela técnica en Córdoba: sentidos y estrategias de la transformación educativa. Un análisis de mesonivel. Córdoba: Edit. FFyH.

ALTERMAN, N. (2005). El Ciclo de Especialización en la escuela secundaria de Córdoba: currículum, organización escolar y gestión directiva. Córdoba: Informe SECyT - UNC.

BREZINSKI, M. A. (2011). O novo modelo para a Educação Profissional e Tecnológica e a Avaliação Institucional: efeitos das Políticas Públicas sobre a configuração do Instituto Federal de Santa Catarina. Dissertação de Mestrado. Brasil: UNICAMP.

BREZINSKI, M. A \& AGUILAR, L. (2013). Efeitos das políticas públicas de educação profissional e de avaliação institucional na configuracão do Insituto Federal de Santa Catarina. Em: AGUILAR, L. \& KASCHNY SCHNEIDER, M. Trajetória de educação profissional e tecnológica. Vol. I. Brasil: Publicação do IFSC.

BRIASCO, I. (2008). ¿Hacia dónde va la educación técnico profesional en América latina?. Argentina: Fundación UOCRA.

-(2008).Tendencias de la educación técnico profesional en América latina. IIPE-

UNESCO. París: Mimeo. 
(2005).Formación Profesional y Capacitación para la Integración y Competitividad. Argentina: OEI - Buenos Aires.

BRIASCO, I. \& VALDES T. (2000). La educación técnico profesional en Iberoamérica. En: Serie Educación Comparada. Cuaderno N8. España: OEI-Madrid.

BRIASCO, I. \& VARGAS F. (2002). La implementación de sistemas por competencias: impacto en el modelo de gestión organizacional. Experiencias y estrategias para la acción. Montevideo: CINTERFOR-OIT/OEI.

CARVALHO, J. (2008). História, trabalho e educação: a educação profissional no Brasil (EPDB - Poços de Caldas - MG). Dissertação de Mestrado. Faculdade de Educação da Universidade Estadual de Campinas. Brasil: UNICAMP.

CIAVATTA, M. y RAMOS, M. (2012). A "era das diretrizes": a disputa pelo projeto de educação dos mais pobres. Revista Brasileira de Educação v. 17 n. 49. jan.-abr.

CONÇALVES, P. (2001). Formação do trabalhador e ensina profissional: Escola Profissional Masculino Rio Claro. Dissertação de Mestrado. Faculdade de Educação da Universidade Estadual de Campinas. Brasil: UNICAMP.

COSTA GOMES, C. (2008). Democratização e financiamento da educação profissional: uma provocativa experiencia. Ensaio: aval. pol. públ. educ., Rio de Janeiro, v. 16, n. 59, p. 179-194, abr./jun.

CREMONESE, B. (2012). Análise política do Programa Nacional de Integração da Educação Profissional com a educação básica na modalidade de educação de jovens e adultos - PROEJA Dissertação de Mestrado. Faculdade de Educação da Universidade Estadual de Campinas. Brasil: UNICAMP.

DA CRUZ, L. (2008). Bento Quirino e COTUCA: os passos do Ensino Profissional em Campinas. Dissertação de Mestrado. Faculdade de Educação da Universidade Estadual de Campinas. Brasil: UNICAMP.

DA SILVA, M. (2011). A política de integração curricular no âmbito do PROEJA: entre discursos, sujeitos e práticas. Ensaio: aval. pol. públ. educ., Rio de Janeiro, v. 19, n. 71, p. 307-326, abr./jun.

DELPHINO, F. (2010). Uma reflexão sobre o percurso histórico da educação profissional pública Brasil - Argentina. En: Miranda, E. \& Bryan, N. (2011). (Re) pensar la educación pública. Un aporte desde Argentin y Brasil. Argentina: Edit. FFyH - UNC.

-(2009). A Educação Profissional. Contraponto entre as políticas educacionais e o contexto do mundo produtivo. Brasil: Edit. Icone.

DUSSEL, I \& PINEAU, P. (2003). De cuando la clase media entró al paraíso: la educación técnica estatal en el primer peronismo. En: Puiggrós A. (Dir.) \& Carli, S. (Coord.) Discurso pedagógicos e imaginario social en el peronismo (1945 - 1955), Historia de la Educación Argentina. Tomo VI. Ed Galerna, Buenos Aires. 1ra. Reimp. 
ESLABÃO, L. (2006). A construção de um currículo por competências: o caso do Curso Técnico em Sistemas de Telecomunicações do CEFET -Dissertação (Mestrado em Educação). Brasil: Universidad Federal de Pelotas.

FINNEGAN, F. (2006). Reseña de políticas educativas de articulación de la educación media con la formación para el trabajo en América Latina. Los casos de Colombia, Uruguay, México, Brasil y Chile. Informe Final. Buenos Aires: IIPE-UNESCO.

FERRETTI, C. (2009). A reforma do ensino técnico da década de 1990: entre a proposta e a prática. Revista Brasilera de Estudios Pedagógicos, Brasília, V. 90, N. 224, p. 11-31, jan./abr.

FERREIRA, M. \& PAISSÉ, S. (2003). La formación profesional en el MERCOSUR. Montevideo: OIT-CINTERFOR.

FRIGOTTO, G. (2007). A relação da educação profissional e tecnológica com a universalização da educação básica. Revista Educ. Soc., Campinas, vol. 28, n. 100 Especial, p. 1129-1152, Brasil.

FRIGOTTO, G. y CIAVATTA, M. (2003). Educação básica no brasil na década de 1990: subordinação ativa e consentida à lógica do mercado. Educ. Soc., Campinas, vol. 24, n. 82, p. 93-130. Brasil.

FRIGOTTO, G., CIAVATTA, M y RAMOS, M. (2005). A política de educação profissional no governo Lula: um percurso histórico controvertido. Revista Educ. Soc., 26 (92), 1087-1113.

GALLART, M.A. (2006). La escuela técnica industrial en Argentina: ¿un modelo para armar? Montevideo: OIT/CINTERFOR.

-(2004). Habilidades y competencias para el sector informal de América Latina: una revisión de la literatura sobre programas y metodologías de formación. Montevideo: Boletín CINTERFOR. N 155.

(2003). La reforma de la educación técnica en la Argentina durante los años noventa. Modelos, alcance de la implementación y balance actual. En: Gallart, Miranda Oyarzun, Peirano \& Sevilla. Tendencias de la Educación Técnica en América Latina. Estudios de caso en Argentina y Chile. París: IIPE - UNESCO. 33-149 pp.

Oyarzun, Peirano \& Sevilla (2003). Tendencias de la Educación Técnica en América Latina. Estudios de caso en Argentina y Chile. París: IIPE - UNESCO.

(2002). 20 años de educación y trabajo. Montevideo: OIT/CINTERFOR.

GARCÉS, L. (2007). La formación para el trabajo en el sistema educativo. Una mirada histórica. In: GARCÉS, L (Comp.). ¿De la escuela al trabajo? Buenos Aires: Ediciones del Signo.

GARMENDIA ARIGÓN, M. (2000). Legislación comparada sobre formación profesional: una visión desde los convenios de la OIT. Montevideo: CINTERFOR/OIT. 
GONCALVES (2001) Formação do trabalhador e ensino profissional : a Escola Profissional Masculina de Rio Claro. Tesse Mestre em Educação. Brasil: UNICAMP.

GUIMARÃES, E. (2008). Política de Ensino Médio e Educação Profissional: discursos pedagógicos e práticas curriculares. Tese (Doutorado em Educação) - Centro de Educação. Recife: Univ. Fed. de Pernambuco.

IBARROLA, M. (Coord.) (2002). Desarrollo local y formación: hacia una mirada integral de la formación de los jóvenes para el trabajo. Montevideo: OIT/Cinterfor.

IBARROLA, M. \& GALLART, M. A. (1994). Democracia y productividad: desafíos de una nueva educación media en América Latina. Santiago de Chile: IIPE-UNESCO.

JACINTO, C. (2010). Reformulaciones recientes acerca de la formación para el trabajo en la educación secundaria general. En: ALMANDOZ, M. R. (et.al.). Educación y trabajo: articulaciones y políticas. Buenos Aires: IIPE - UNESCO.

(2004) ¿Educar para qué trabajo? Discutiendo rumbos en América Latina. Buenos Aires: IIPE-UNESCO.

JACINTO, C. \& MILLENAAR, V. (2004). Las relaciones entre escuelas y empresas: un camino con nuevos desafíos en América Latina. En: Boletín redEtis $\mathrm{N}^{\circ}$ 7. Buenos Aires: IIPE - UNESCO.

JIMÉNEZ BECERRA, A. (2006). El estado del arte en la investigación en ciencias sociales. En: JIMENÉS BECERRA, A. \& TORRES CARRILLO, A. (Comp.). La práctica investigativa en ciencias sociales. Departamento de Ciencias Sociales. Universidad Pedagógica Nacional. Colombia.

MATURO, Y. \& RUBIO, A. (2008) La transformación educativa en las ex escuelas técnicas de Córdoba (1996 - 2005): una mirada desde el currículum. Trabajo Final de Licenciatura en Ciencias de la Educación, UNC. Disponible en: http://hdl.handle.net/11086/552

MENDES, L. (2005). Políticas Públicas e a Pedagogia das Competências na Educação Profissional: a trajetória do ensino profissionalizante de nível técnico no Brasil e no Estado de São Paulo. Tese de Doutorado. Faculdade de Educação da Universidade Estadual de Campinas. Brasil: UNICAMP.

MELO, S. (2009). Trabalho e conflito docente: experiências em escolas de educação profissional no Brasil e na Argentina. Tese de Doutorado. Faculdade de Educação da Universidade Federal de Minas Gerais. Brasil: UNICAMP.

MERCOSUR (2001). Informe Mercado de trabajo. Observatorio del mercado de trabajo del MERCOSUR. del MERCOSUR.

(2005). Informe Mercado de Trabajo. Observatorio del mercado de trabajo (2011). Informe Mercado de Trabajo. Observatorio del mercado de trabajo del MERCOSUR. 
(2012). Informe sobre Empleo juvenil en el MERCOSUR.

MESSINA, G.; WEINBERG, P. \& IRIGOIN, M. (1996). La educación técnica y la formación profesional. Chile: UNESCO..

MIRANDA, E. (2011) Una "caja de herramientas" para el análisis de políticas educativas. La perspectiva de los ciclos de las políticas (Policy Cycle Approach). En Miranda, E y Bryan, N. (2011) (Edit.) (Re) Pensar la Educación Pública. Aportes desde Argentina y Brasil. Editorial de la Facultad de Filosofía y Humanidades/UNC, Córdoba.

NEVES. C. (2008). Las organizaciones internacionales y la evaluación de los sistemas de educación y formación: análisis crítico y comparativo. Revista Europea de Formación Profesional $\mathrm{N}^{\circ}$ 45, Vol 3. Luxemburgo: CEDEFOP.

NOVICK, M., BARTOLOME, M., BUCETA, M. \& Otras (1998) Nuevos puestos de trabajo y competencias laborales. Un análisis cualitativo del sector metalmecánico Montevideo: OIT/Cinterfor.

ORTIGARA, C. (2012) Reformas educacionais no período Lula (2003-2010): implementação nas instituições federais de ensino profissional. Tese de Doutorado. Faculdade de Educação da Universidade Estadual de Campinas. Brasil: UNICAMP.

PINEAU, P. (1997). La vergüenza de haber sido y el dolor de ya no ser: los avatares de la educación técnica entre 1955 y 1983. En: Puiggrós, A. (Coord.) Historia de la Educación Argentina. Dictaduras y utopías en la historia reciente de la educación Argentina (1955 1983). Tomo VIII. 1ra Reimp. Buenos Aires: Ed Galerna.

PUIGGRÓS, A. \& BERNETTI, J. L. (1993). Peronismo: cultura política y educación (1945 - 1955). En PUIGGRÓS A. (Dir.). Historia de la Educación Argentina. Tomo VI. Buenos Aires: Ed Galerna.

POLI, C. (1999). Ensino médio profissionalizante: quem o quer? a quem ele serve? Dissertação de Mestrado. Faculdade de Educação da Universidade Estadual de Campinas. Brasil: UNICAMP.

SILVA, R. (2004) Escola Profissional Mixta "Cel. Francisco Garcia": as relações entre Educação e trabalho no capitalismo periférico (1931-1936). Dissertação de Mestrado. Faculdade de Educação da Universidade Estadual de Campinas. Brasil: UNICAMP.

SOBREVILA, J. A. (1995) La educación técnica argentina. Serie Estudios. Academia Nacional de Educación. Buenos Aires.

SPINOSA \& TESTA (2009). L'enseignement professionnel en Argentine. Entre volontarisme et isolement dans la recherche d'un pays posible. En: Formation Emploi, $\mathrm{N}^{\circ}$ 107, págs. 9-24.

STEINKOL W. y CROCE A. (2001). Los talleres pre-ocupacionales: evolución e impacto. En: II Congreso Internacional de Educación, Escuela Normal Superior Dr. Alejandro Carbó. Córdoba. 
TACCARI, D. (2011). Los sistemas internacionales de indicadores educativos en Latinoamérica. En: KISILEVSKY, M. \& ROCA, E. (Coord.). Indicadores, metas y políticas educativas. Colección Metas Educativas 2021. España: OEI.

TELLO, C. \& MAINARDES, J. (2012). La posición epistemológica de los investigadores en Política Educativa: debates teóricos en torno a las perspectivas pos-estructuralista, neomarxista y pluralista. Revista Archivos Analíticos de Políticas Educativas, v. 20, n. 9, p. 1 31, mar.

TESTA, J. \& SANCHEZ, P. (2003). "Una perspectiva crítica y alternativa en relación con la formulación, diseño e implementación de los Trayectos Técnicos Profesionales”, en Coloquio: a diez años de la Ley Federal de Educación. Educación para todos, Ceil-Piette del CONICET, Área Educación y Trabajo.

VELASCO, C. (2007). Educación técnica y formación profesional en América latina y el Caribe. Santiago de Chile: OREALC/UNESCO.

(2005). La educación técnico profesional de nivel medio en 7 países de América Latina. Aproximaciones a un estado del arte. Chile: UNESCO.

\section{Notas}

${ }^{1}$ En este trabajo se recuperan avances de la Tesis Doctoral en Ciencias de la Educación (en curso), titulada: "Del discurso a la práctica: la trayectoria de la política educativa para la Educación Técnico Profesional de nivel medio en las escuelas de orientación industrial de Argentina y Brasil a partir del 2003”; radicada en el Centro de Investigaciones María Saleme de Burnichon de la Facultad de Filosofía y Humanidades de la Universidad Nacional de Córdoba, Beca de postgrado CONICET 2014- 2019.

${ }^{2}$ Profesora y Licenciada en Ciencias de la Educación (UNC). Especialista en Ciencias Sociales (FLACSO). Profesora Adscripta en la Escuela de Ciencias de la Educación (UNC). Profesora Asistente dedicación Semiexclusiva en la Escuela de Ciencias de la Información (UNC). Participa del Grupo de Investigación "Estudios sobre políticas educativas" (UNC). E-mail: yaninamaturo@ hotmail.com

3 Durante la década de los '90 las demandas de mayor calificación vinculadas a las transformaciones tecnológicas, a la competitividad y a la globalización se evidenciaron en el desarrollo de políticas para la ETP que se nutrieron de argumentaciones económicas; impregnando de racionalidad instrumental las estrategias que expresaba el discurso político para la modalidad (Almandoz, 2010). Tanto en Argentina como en Brasil, este proceso consistió en la introducción de modificaciones tanto en la organización de la oferta como en el currículum, que estuvieron orientadas a proponer un conjunto amplio de competencias generales consideradas como transversales al nivel de enseñanza y de acuerdo a los requerimientos del mercado (Jacinto, 2010). Iniciada la primera década del siglo XXI el nuevo contexto político económico y los "efectos no deseados de las reformas educativas anteriores (década de los '90)" marcaron un nuevo rumbo en el debate acerca de las políticas y estrategias para la educación y, en particular, para la ETP (Frigotto, 2007; Gallart, 2006).

${ }^{4}$ Programa de Integração da Educação Profissional Técnica de Nível Médio na Modalidade de Educação de Jovens e Adultos.

${ }^{5}$ Organización Internacional del Trabajo - Centro Interamericano para el Desarrollo del Conocimiento en la Formación Profesional.

${ }^{6}$ Comisión Económica para América Latina.

${ }^{7}$ Instituto Internacional de Planeamiento de la Educación - Organización de las Naciones Unidas para la Educación, la Ciencia y la Cultura. 


\footnotetext{
${ }^{8}$ Esta idea será ampliada en el próximo apartado.

${ }^{9}$ Se retoma el concepto de "discurso" de Stephen Ball, citado por Miranda (2011).

${ }^{10}$ Organización de las Naciones Unidas.

${ }^{11}$ Oficina Regional de Educación para América Latina y el Caribe.

${ }^{12}$ Programa Regional de Educación para América Latina y el Caribe.

${ }^{13}$ Cabe mencionar asimismo que paralelamente, con el propósito de acompañar los cambios que se proponen, estos organismos han implementado un amplio programa de actividades con la intención de apoyar el logro de las metas/objetivos propuestos (coordinación de los programas de acción compartida, la captación de fondos y recursos para el desarrollo de los proyectos emprendidos, la evaluación y el seguimiento de las metas/objetivos propuestos) y colaborar mutuamente en su aplicación.
}

Recebido: julho/14 Aprovado: agosto/14 